\title{
Experimental and Numerical Approaches for Reliability Evaluation of Electronic Packaging*
}

\author{
Soon-Bok LEE** and Jae-Won JANG** \\ ${ }^{* *}$ Department of Mechanical Engineering, KAIST \\ 291 Daehak-ro, Yuseong-gu, Daejeon 305-701, Republic of Korea \\ E-mail: sblee@kaist.ac.kr
}

\begin{abstract}
There are two major methods, experimental and numerical approaches, for dealing with the strain and stress analysis essential to reliability assessment of electronic packaging. Both approaches are mutually complementary in view point of their assessment capability. In this paper, experimental and numerical simulation results for analyzing some reliability issues of electronic packages were reviewed, and the role of both approaches were discussed.
\end{abstract}

Key words: Experimental Method, Numerical Analysis, Reliability Evaluation, Electronic Packaging, Fatigue

\section{Introduction}

There are lots of demands and supplies for high performance and multi-functional electronic devices which could enrich our lives. Nowadays, we could easily see novel products day by day due to fast development in technology. Therefore, the time for products development including their reliability prediction becomes shorter. Consequently, it can cause various reliability problems of the products, and may lead to social damage, such as not only financial loss but also the loss of human life. Therefore, reliability assessment of the products is undoubtedly important and researchers should put in a great deal of effort on resolving these problems.

Over the decades, experimental and numerical approaches have been used to address reliability issues of electronic packaging. With advanced measurement method, it is possible to observe sub-micron-scale deformations. The strain or stress evaluation of tiny and complex structures through numerical approaches is also realized due to the enhancement of computational models and computing hardware.

There are many reliability issues in electronic packaging and tremendous studies were conducted. Some of them employed the experimental technique as their methodology. Characterization of mechanical properties and fatigue life estimation of solder joints- most commonly used interconnection materials in electronic packaging- were conducted. ${ }^{(1),(2),(3)}$ Kim et al. ${ }^{(4)}$ developed the impact tester using an electromagnetic actuator and evaluated the impact fatigue life of solder joints. Copper diffusion phenomenon in copper wire bonding was also experimentally studied. ${ }^{(5)}$ One of the key reliability issues is the effect of moisture concentration and absorption in packaging structures. Hygroscopic swelling behavior of molding compounds and plastic packages was investigated with a moiré interferometry. ${ }^{(6)}$ Numerical simulation, such as finite element method (FEM) has been also widely utilized as a methodology for reliability assessment of electronic packages. Parametric studies for reliability evaluation of through-silicon vias (TSVs) for 
3-dimensional (3D) interconnection were conducted using FEM. (7), (8) In addition, combination of experimental and numerical approaches was also adapted for various reliability evaluations of electronic packages. Residual stress of resin-molded integrated circuit (IC), which is one of the critical factors in their reliability, was evaluated. ${ }^{(9)}$ Humidity effects on the delamination in a flip chip using anisotropic conductive adhesive films $(\mathrm{ACFs})^{(10)}$ and the solder reflow crack in a quad flat package ${ }^{(11)}$ were evaluated. Hygrothermal properties of ACF package were experimentally obtained and moisture concentration distribution was obtained using FEM by Yoon et al. ${ }^{(12)}$

In this paper, experimental methods and numerical approaches are briefly reviewed, which were performed in the Computer Aided Reliability Evaluation (CARE) laboratory in KAIST for reliability evaluation of electronic packaging. Especially, some of the reliability issues including low and high cycle fatigue, ${ }^{(13)}$ creep and relaxation behavior, ${ }^{(14)}$ delamination, ${ }^{(15)}$ and warpage ${ }^{(15),(16)}$ were summarized in this paper. The main focus in this paper is how the experimental and numerical approaches have been employed and compensated each other in each topic. In addition, the development trend of electronic packaging can be observed in the specimens used in following chapters. Finally, the role of the experimental and numerical approaches in the future is discussed and further works related to reliability evaluation of electronic packaging using both approaches in CARE group are also introduced. ${ }^{(17),(18)}$

\section{High and Low Cycle Fatigue}

High and low cycle fatigue tests for evaluating the reliability of the solder bump performed by Lee et al. ${ }^{(13)}$ were introduced in this chapter. A small-sized electromagnetic type fatigue tester was developed, and the loading fixture for the specimen was designed for torsional fatigue loading as shown in Fig. 1. The specimens are made with two pieces of printed circuit boards (PCBs) connected by four ball grid array (BGA) type solder joints. Two types of specimens were made with the same shape but different thickness of PCBs, i.e. $1.2 \mathrm{~mm}$ and $1.6 \mathrm{~mm}$ PCBs. They performed fatigue tests the leaded surface-mount components in the torsional loading configuration at the frequency of $40 \mathrm{~Hz}$.

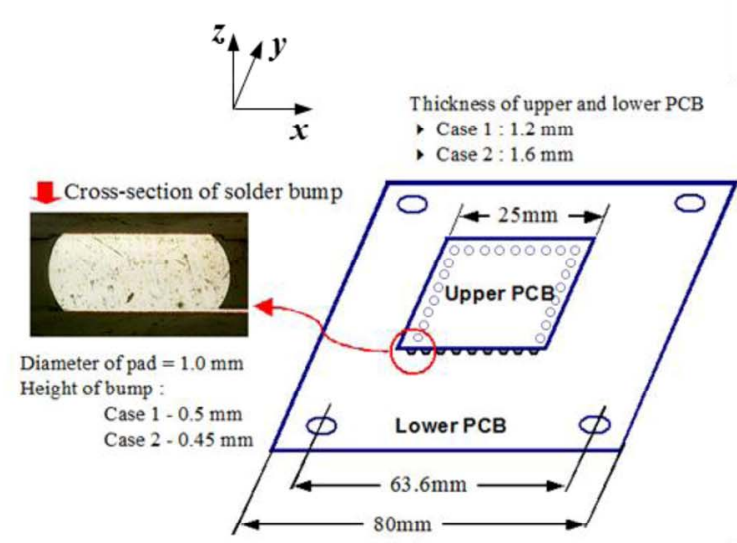

(a)

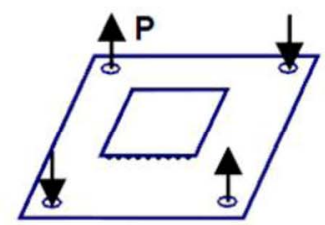

(b)

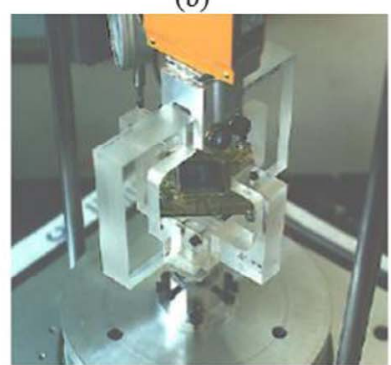

(c)

Fig. 1. (a) The specimen; PCB/32 solder bumps/PCB assembly. (b) Schematic loading configuration. (c) A photo of the loading fixture (adapted from (13)).

To define the failure of the specimens, the change in resistance was recorded. A typical resistance vs. cycles curve is shown in Fig. 2. As shown in Fig. 2, there are two abrupt increases in the resistance. The cross section of a cracked solder bump after the first abrupt 
resistance change is shown in Fig. 2(a). The crack is propagated through the surface between the solder bump and the copper pad on the PCB caused the second abrupt resistance change as depicted in Fig. 2(b). That is, the second abrupt change means the final separation between the copper pad on the PCB and the solder bump located at the corner of the specimen. For a conservative life prediction, the failure life of the specimens was defined by the first rapid increase in the resistance.

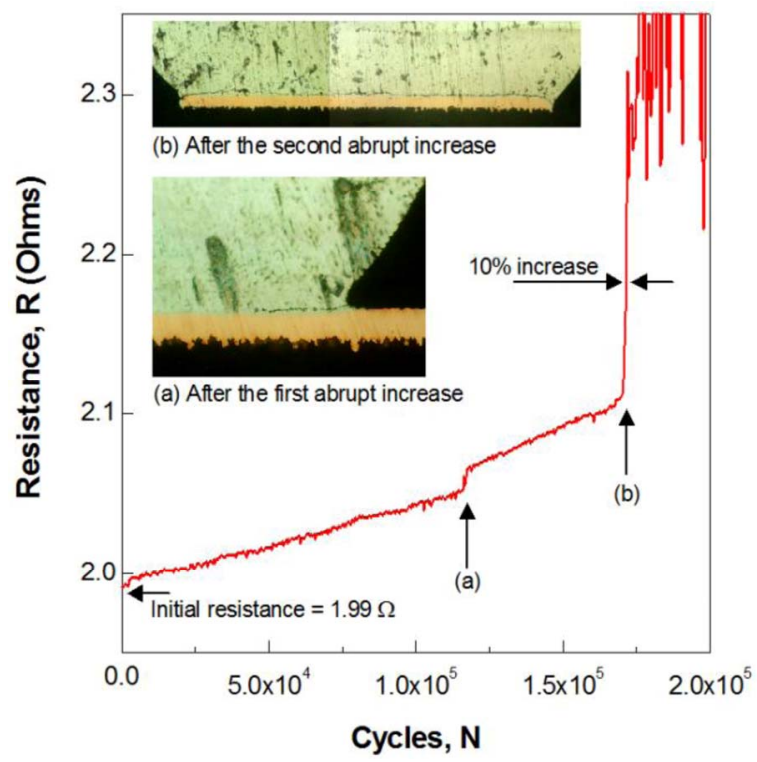

Fig. 2. A typical resistance versus cycles result (adapted from (13)).

During the high cycle fatigue tests, it is difficult to measure the stress distribution experimentally. However, the investigation of stress distribution of the solder joint is essential to analyze the damage parameter. To compensate the limitation of experimental approach, elastic-plastic finite element analysis (FEA) was adapted. That is, to obtain the stress distribution of the solder joint, FEAs were performed and a typical result is shown in Fig. 3.
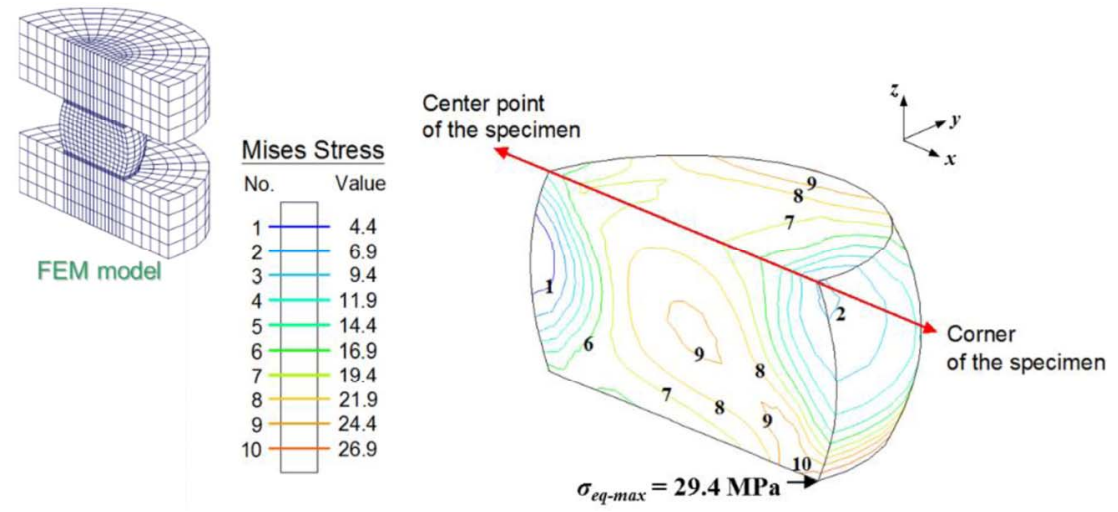

Fig. 3. Von Mises stress contour of the solder joint obtained by FEA.

Figure 4(a) shows the high cycle fatigue test results. As shown in the Fig. 4(a), the load amplitude is not a good damage parameter of solder joints regardless of the thickness of the PCBs. On the other hand, the maximum equivalent stress amplitude widely used to predict yielding of materials obtained from the FEAs and the failure cycles show linear relationship in Fig. 4(b) irrespective to the thickness of the PCBs. 


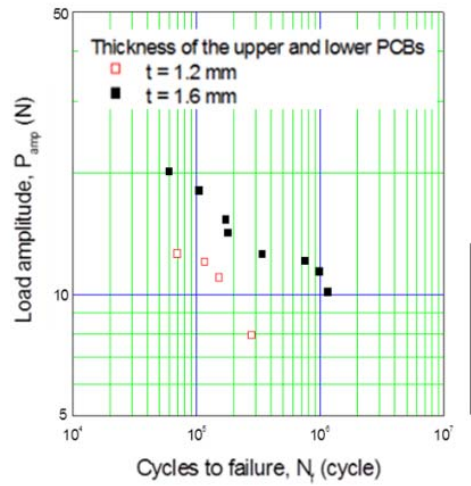

Load amplitude vs. failure cycles

(a)

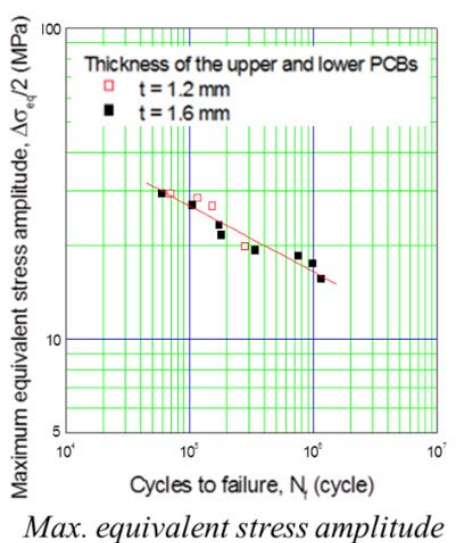

vs. failure cycles

(b)

Fig. 4. (a) Load amplitude vs. failure cycles; (b) Maximum equivalent stress amplitude vs. failure cycles (adapted from (13)).

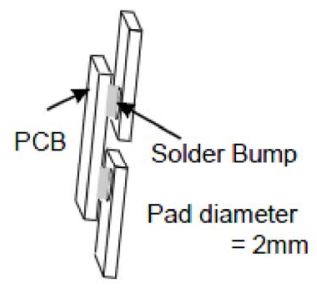

(a)

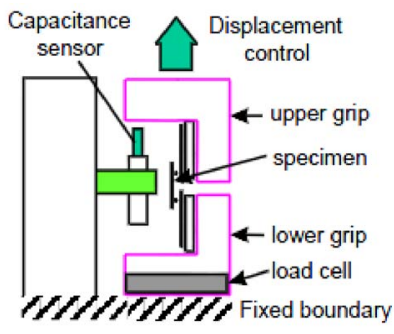

(b)

Fig. 5. Configuration of the specimen and loading fixture; (a) The specimen having the two BGA-type solder bumps; (b) Loading fixture of low cycle fatigue test (adapted from (13)).

A micro-mechanical tester was developed and the specimens are three pieces of PCBs connected by two solder joints as shown in Fig. 5(a). The loading fixture for the specimen was designed to be suitable for fatigue loading as seen in Fig. 5(b). The effect of solder joint shape on the fatigue life was investigated through the fatigue test with different solder shape specimens.

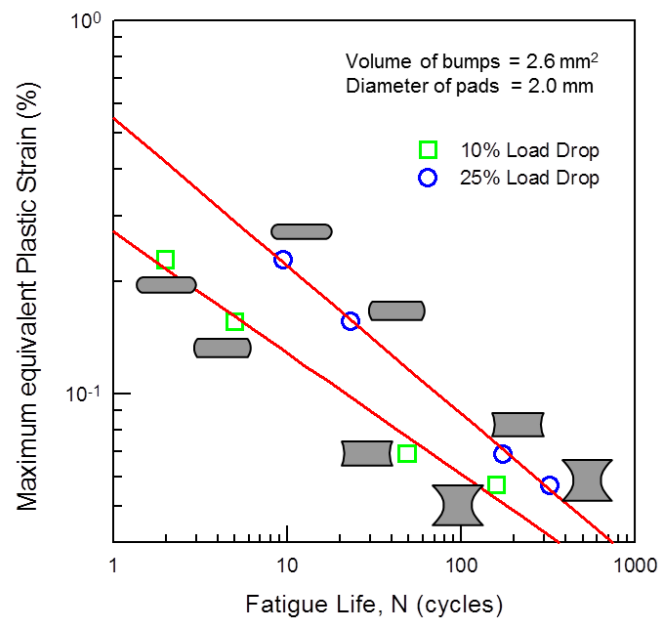

Fig. 6. Maximum equivalent plastic strain versus fatigue life defined by $10 \%$ and $25 \%$ load drop (adapted from (13)). 
Fatigue tests were performed in a low-cycle displacement-controlled mode in which the joints were subjected to a fully reversed ramp cycle at the constant frequency of $1 \mathrm{~Hz}$. The correlation of fatigue life defined by the crack initiation cycles versus maximum equivalent plastic strain obtained by FEA is shown in Fig. 6. The fatigue life curve of solder bumps with various shapes becomes straight lines.

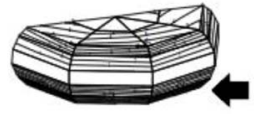

(a)
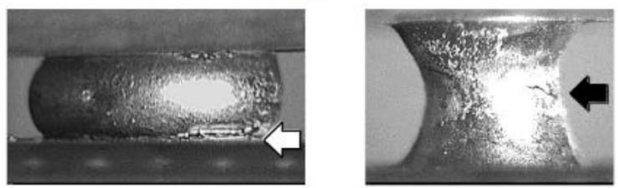

(b)

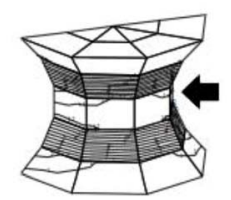

Fig.7. The prediction of crack location from the finite element analysis: (a) Distributions of equivalent plastic strain; arrow indicates high strain location, (b) Photography of the fatigue failure on the solder bumps (adapted from (13)).

Figure 7(a) shows the locations where the maximum equivalent plastic strain occur in two distinguished shape of solder bump obtained from FEAs marked by arrows. The experimental failure locations are shown in Fig. 7(b), and the locations of crack initiation are in good agreement with those where the maximum equivalent plastic strains occurs respectively.

\section{Creep and Relaxation}

Ham et $a l .{ }^{(14)}$ observed the creep and relaxation behaviors of a wafer-level chip scale package (CSP) assembled to a PCB under thermal loadings. Figure 8 shows the cross-sectional picture of the specimen. To observe the deformations of the specimen using moiré interferometry, the specimen grating was replicated on the target surface of the specimen.

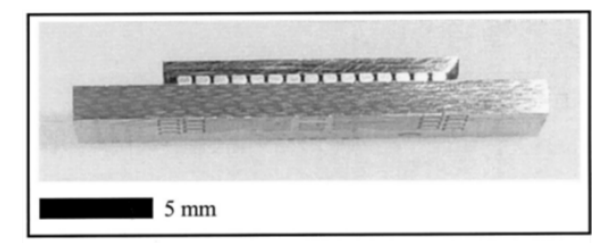

(a)

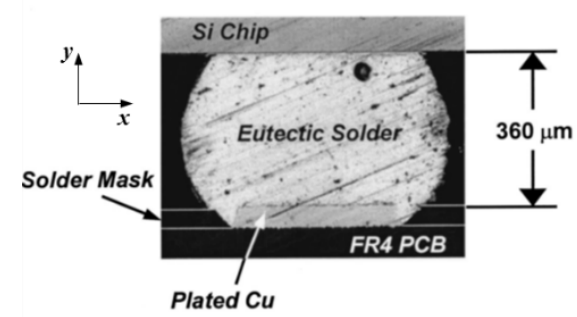

(b)

Fig. 8. Specimen for moiré interferometry study: (a) specimen cut from CSP assembly, providing cross section having 15 solder bumps; (b) cross section of the solder bump of CSP assembly (adapted from (14)). 


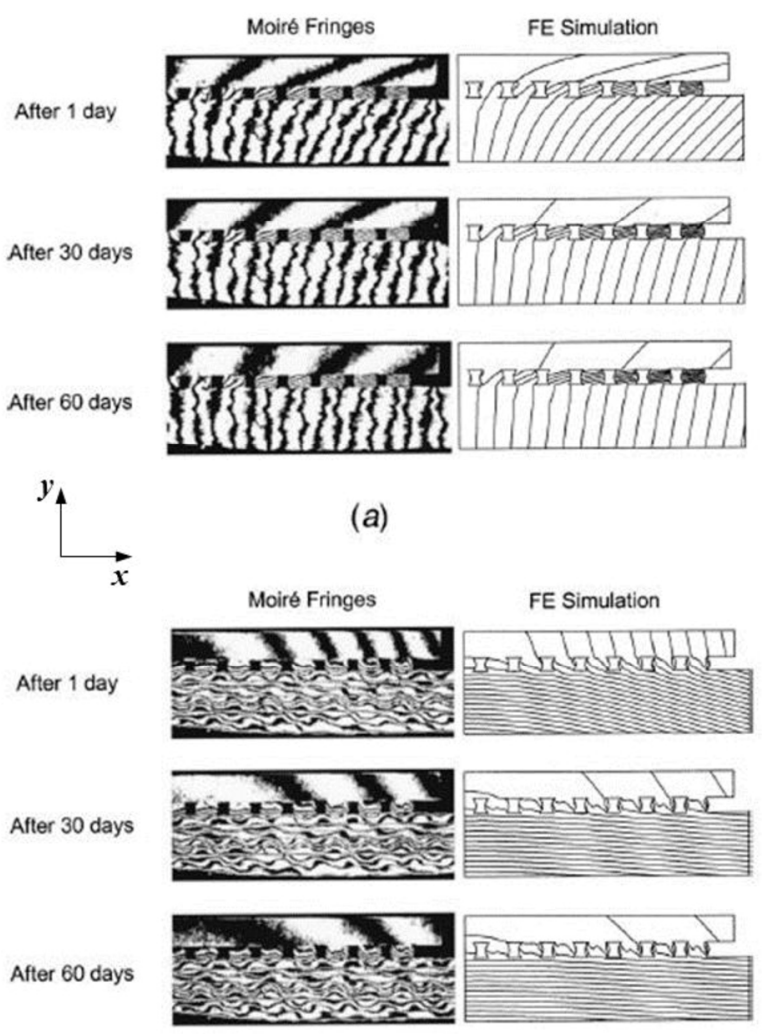

(b)

Fig. 9. Moiré fringes and FEA simulation of the right half of a CSP assembly corresponding to the isothermal loading case from $100{ }^{\circ} \mathrm{C}$ to room temperature $(\Delta T=$ $\left.-75{ }^{\circ} \mathrm{C}\right)$ : (a) $\mathrm{U}$ ( $x$-directional) displacement field; (b) $\mathrm{V}$ ( $y$-directional) displacement (adapted from (14)).

From the moiré experiments, displacement fields of the CSP assembly were obtained and the results are shown in Fig. 9. FEA results corresponding to each experimental result are also shown in Fig. 9.

As shown in Fig. 9, remarkable difference exists between the deformation of the Si chip and that of the PCB because of the difference between their coefficients of thermal expansion (CTEs). At the ends of the chip, the relative displacement reaches its maximum value, which causes the highest stress and strain in the rightmost solder bump.

Both experimental results and numerical results show good agreement in the global deformation tendency. However, it is difficult to simulate the real deformation of the component material of the assembly observed by using moiré experiments. On the other hand, it is possible to simulate the interest model under extreme and various conditions by using FEA simulation, but limited to the experiments.

\section{Delamination and Warpage}

In this chapter, life prediction models for flip-chip package with anisotropic conductive adhesive (ACA) interconnection proposed by Yang et al. ${ }^{(15)}$ and Park et al. ${ }^{(16)}$ were introduced. Compared to solder ball interconnection used for the specimen in previous chapters, ACA interconnection as shown in Fig. 10 can provide much higher density interconnection by decreasing its pitch. Furthermore, fabrication process can be simplified by adapting wafer-level packaging. 


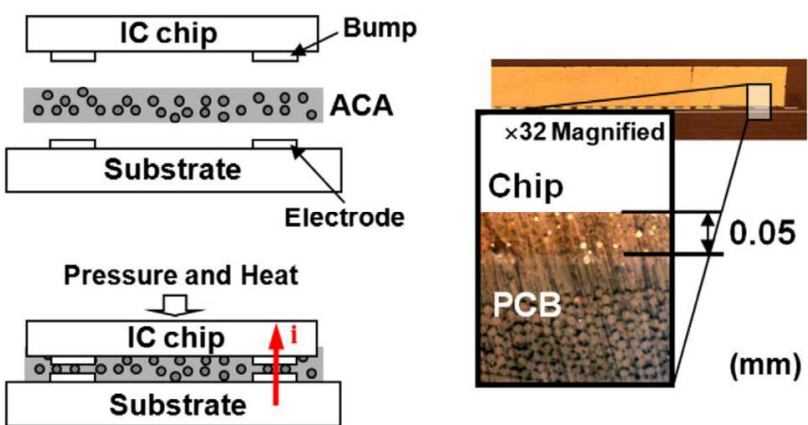

Fig. 10. Schematic and real cross section image of an ACA type flip-chip (adapted from (15)).
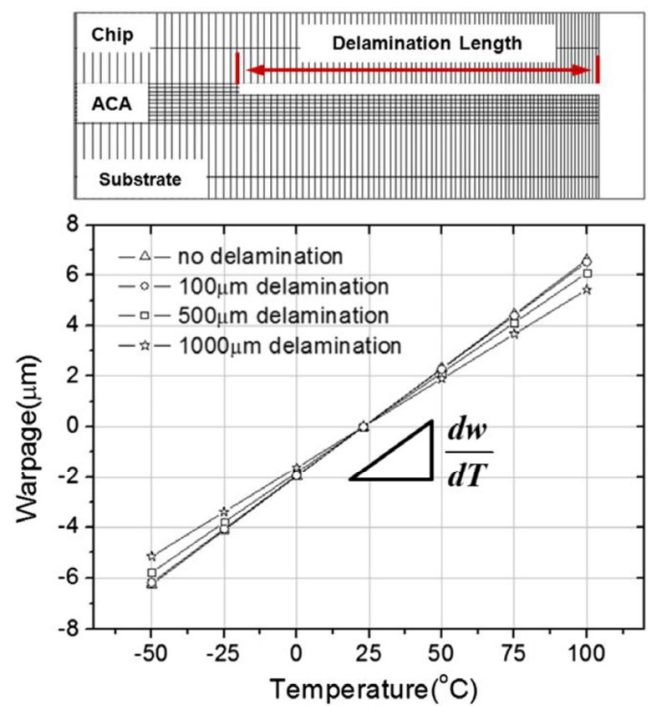

Fig. 11. Numerical estimation of chip warpage behavior with different delamination length. Decrease in $d w / d T$ for longer delamination is apparent (adapted from (15)).

Yang et al. ${ }^{(15)}$ showed in Fig. 11 through FEA that the chip warpage, the maximum relative vertical displacement, variation with respect to temperature $(d w / d T)$ decreased as the length of delamination at the chip edge increased. Experimental verification was performed by measuring $d w / d T$ of a pre-delaminated ACA type package and both experimental data and results of numerical simulation were co-plotted in Fig. 12.

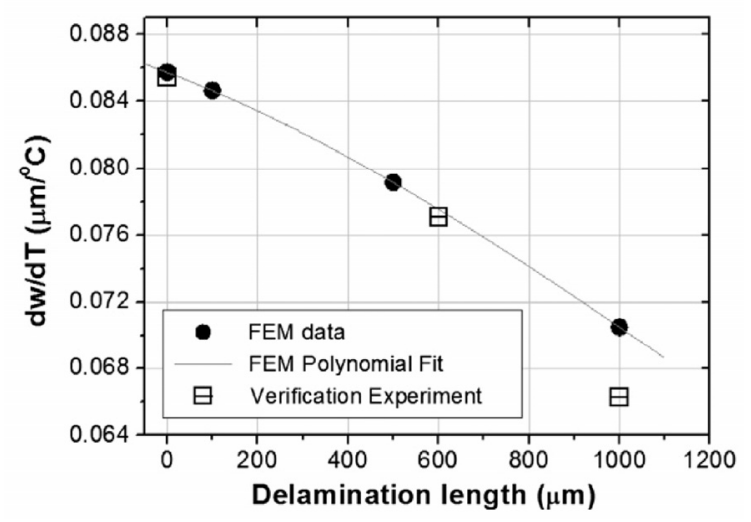

Fig. 12. Relationship between $d w / d T$ and delamination length obtained both numerically and experimentally (adapted from (15)). 


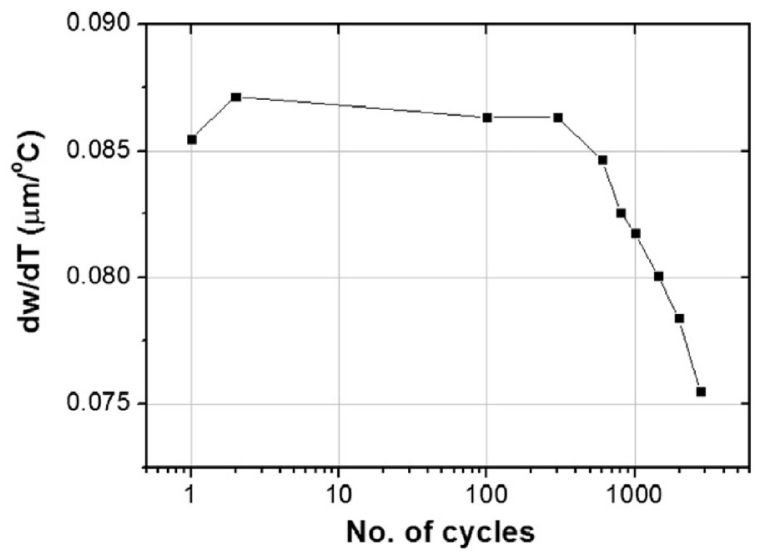

Fig. 13. Change in $d w / d T$ with respect to increase in thermal fatigue cycles $\left(-50{ }^{\circ} \mathrm{C}\right.$ to $+125^{\circ} \mathrm{C}$ ). Measured with in-plane moiré interferometer (adapted from (15)).

Subsequently, they observed $d w / d T$ variation according to increasing thermal fatigue cycles as shown in Fig. 13. Combining this result and the verified relationship between $d w / d T$ and delamination length as shown in Fig. 12 provide delamination vs. number of thermal fatigue cycles as in Fig. 14. It characterizes the initiation and propagation behavior of the ACA type package. An overall lifetime can be predicted by setting a failure criterion of

$$
a \geq a_{c}
$$

where $a$ and $a_{c}$ refer to the delamination length and the critical length to failure, respectively.

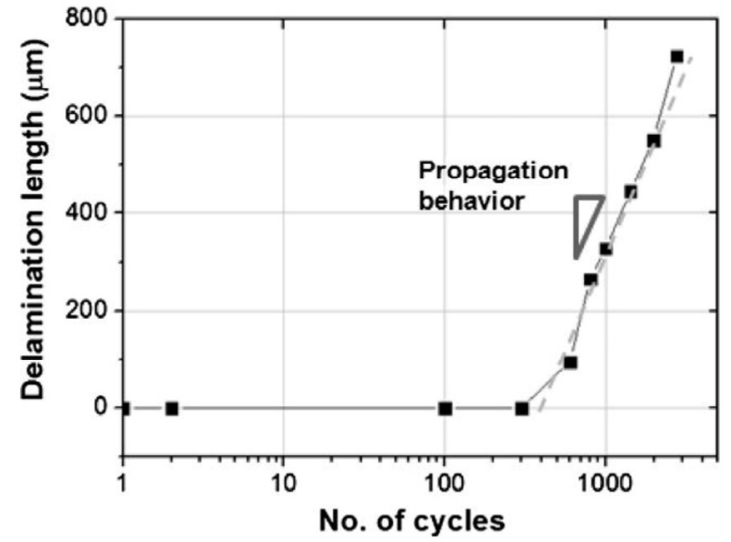

Fig. 14. Change in dw/dT with respect to increase in thermal fatigue cycles $\left(-50{ }^{\circ} \mathrm{C}\right.$ to $+125^{\circ} \mathrm{C}$ ) (adapted from $(15)$ ).

A measurement of $d w / d T$ will provide the status of current damage. In addition, remained life at a given $d w / d T$ can be estimated by taking into account of the propagation behavior and the failure criterion in Eq. (1).

Park et al. ${ }^{(16)}$ proposed thermal damage model for predicting thermal fatigue life of ACA type packages regardless of geometric variations including the presence or absence of fillet and thickness of each components as listed in Table I. Thermal fatigue life under the temperature variation from $-40^{\circ} \mathrm{C}$ to $+125^{\circ} \mathrm{C}$ of each type of specimen were also listed. 
TABLE I. ACF package specimens (adapted from (16)).

\begin{tabular}{ccccc}
\hline \hline Specimen & $\begin{array}{c}\text { Thickness } \\
\text { of chip }(\mu \mathrm{m})\end{array}$ & $\begin{array}{c}\text { Thickness } \\
\text { of PCB }(\mu \mathrm{m})\end{array}$ & Fillet & Life (cycles) \\
\hline S1 & 180 & 120 & - & 14223 \\
S2 & 180 & 550 & - & 15121 \\
S3 & 180 & 980 & - & 4687 \\
S4 & 480 & 120 & - & 11743 \\
S5 & 480 & 550 & - & 11744 \\
S6 & 480 & 980 & - & 2737 \\
\hline $\mathrm{NF}^{1)}$ & 780 & 980 & - & 2533 \\
$\mathrm{WF}^{2)}$ & 780 & 980 & $\mathrm{O}$ & 4665 \\
\hline \hline
\end{tabular}

${ }^{1)} \mathrm{NF}$ : specimen without fillet; ${ }^{2)} \mathrm{WF}$ : specimen with fillet.

They conducted moire experiments for obtaining strain distribution of the specimens. Figure 15 shows the shear strain distribution of the specimens and locally concentrated strains can be easily distinguished.

At this point, it is worthy to note that even though the strain distribution can be obtained by the optical deformation measurement method in their work, there are limitations of optical deformation measurement methods for measuring tiny specimens smaller than the specimens listed in Table I. There are two major alternative ways for obtaining strain distributions of tiny specimens. One is adapting numerical simulation. However, it is widely known that size effect on material properties of a specimen cannot be negligible. Unfortunately, material properties are also influenced by fabrication process. In addition, in most cases, finite element (FE) model and the real electronic packages do not geometrically match each other due to their complex internal structure. This geometric simplification of FE model for reducing computational time and cost should be verified whether it is reasonable or not. It means that it is not easy to calculate the accurate simulation result due to unknown component material properties and geometric complexity. The other way for obtaining strain distribution of tiny specimens is employing alternative methods, such as high resolution deformation measurement method. There are numerous studies related to the issue and some works will be introduced in the next chapter.

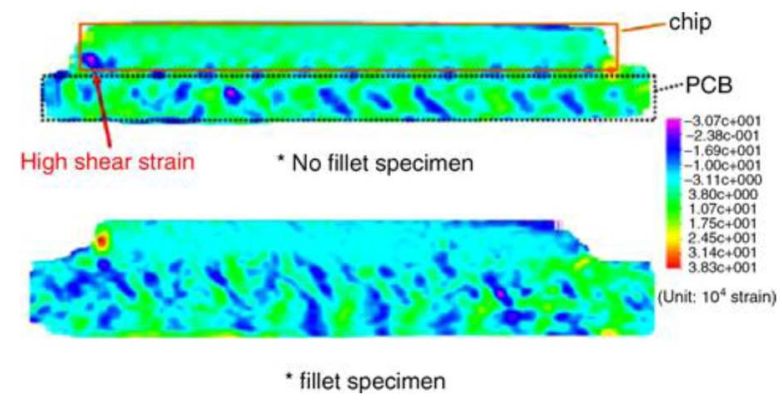

Fig. 15. Shear strain distribution of ACF-type specimens at $25{ }^{\circ} \mathrm{C}$ (adapted from (16)).

The obtained shear strains of each specimen with respect to their thermal fatigue life listed in Table I are plotted in Fig. 16. Application of moderate geometric shape used to reduce the stress concentration, e.g. fillet was shown to be a powerful method for reducing the shear strain. The fillet at the edge of the chip was shown to be useful for increasing the thermal fatigue life. Consequently, low shear strain is shown to improve the reliability of the interconnection layer. 


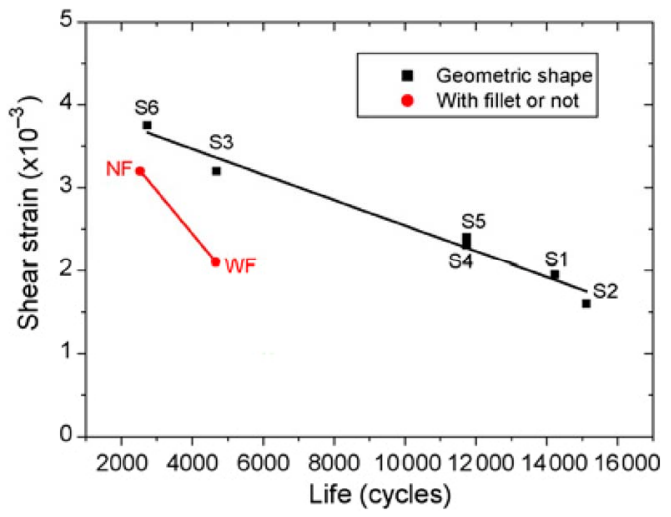

Fig. 16. Relation between thermal fatigue life and shear strain (adapted from (16)).

In order to predict the thermal fatigue life of the ACF-type package, a new damage model that considers the geometric shape and the fillet of the ACF-type package was developed. Park et al. ${ }^{(16)}$ suggested the thermal damage model as described in Eq. (2) by modifying the Morrow energy model widely used in fatigue life prediction.

$$
\Delta W=G \gamma\left(\frac{d w}{d T}\right)
$$

where $G$ is shear modulus and $\gamma$ is shear strain.

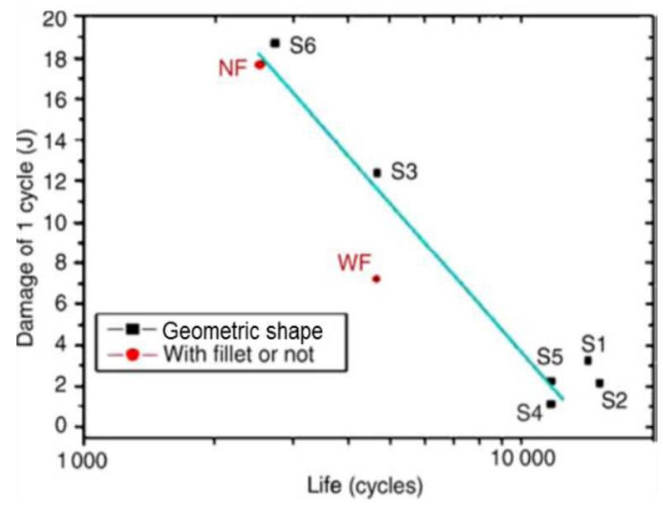

Fig. 17. Relation between thermal life and damage (adapted from (16)).

Figure 17 shows the relationship between the thermal fatigue damage per cycle and the package lifetime. Regardless of the geometric shape and the presence or absence of fillet, the thermal fatigue life is well represented by the proposed damage parameter.

\section{Challenging Issue}

Current works related to reliability evaluation of electronic packages with tiny and complex structure were introduced in this chapter. As described in previous chapter, alternative deformation measurement methods are needed to observe the deformations of the packages which become thinner and finer structure.

Jang et al ${ }^{(17)}$ proposed pseudo-phase-shifting technique which can make AFM moiré method, one of the high resolution microscope moiré methods, applicable for measuring small in-plane deformations by resolving the problems of AFM system. Using the proposed technique, thermally induced deformation of TSV structure was obtained. ${ }^{(18)}$ TSV structure is a key technology for 3D IC integration as shown in Fig. 18. The fine pitch specimen grating with approximately $180 \mathrm{~nm}$ pitch was fabricated by focused ion beam milling. 


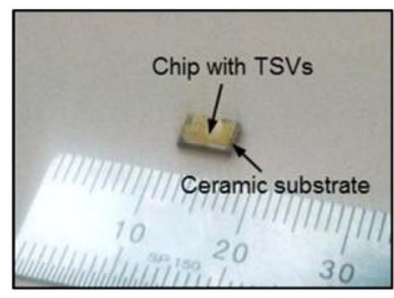

(a)

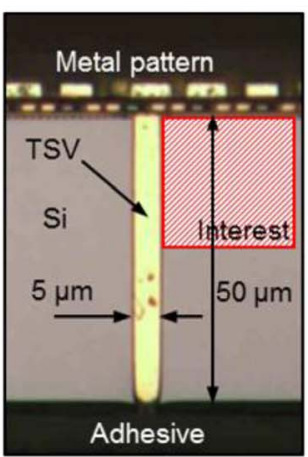

(b)

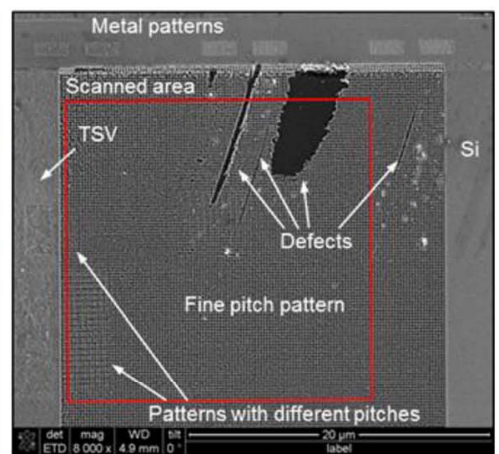

(c)

Fig. 18. (a) TSV specimen, (b) cross-section view of the specimen, (c) scanning electron microscope image after specimen grating fabrication (adapted from (18)).

Figures 19(a) and (b) show V field displacement maps of the region remarked by the rectangular in Fig. 18 before and after applying isothermal loading, respectively.

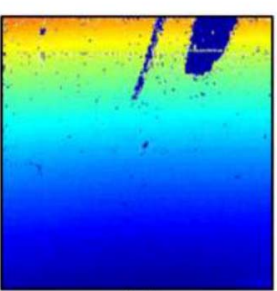

(a)

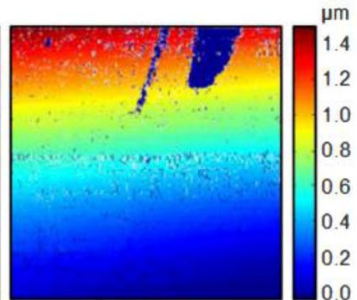

(b)

Fig. 19. V field displacement of the specimen at (a) $31.0 \pm 0.1{ }^{\circ} \mathrm{C}$ and (b) $104.8 \pm$ $0.2{ }^{\circ} \mathrm{C}$, respectively. The size of each image is $20.62 \times 20.57 \mu \mathrm{m}^{2}$ (adapted from (18)).

\section{Conclusion}

Figure 20 illustrates the relationship between the numerical and experimental approaches for the reliability evaluation of electronic packaging described in this paper.

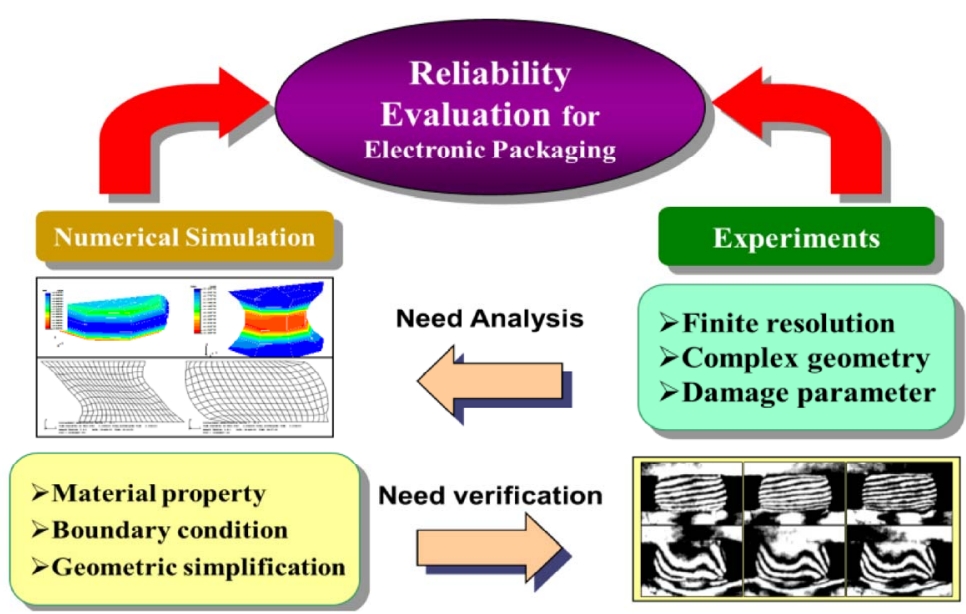

Fig. 20. Relationship between the numerical and experimental approaches.

According to the trend of electronic packages, it can be expected that their size will shrink continuously. It means that the performance of mechanical testing system and the ability, e.g. resolution, field of view, and deformation measurement method should be improved to characterize the packages and assess their reliability. However, only the development of experimental technique is not sufficient in such researches. Since the 
numerical and experimental approaches are mutually complementary, computational models and computing hardware should be also developed for performance enhancement of numerical approach along with the development of experimental technique. Therefore, it is encouraged to use the both approaches with mutual compensation in reliability evaluation of electronic packaging.

\section{Acknowledgement}

This work was supported by the National Research Foundation of Korea (NRF) grant funded by the Korea government (MEST) (No. 2011R1A2A2A02649388).

\section{References}

(1) M. Amagai, M. Watanabe, M. Omiya, K. Kishimoto, and T. Shibuya, Mechanical characterization of Sn-Ag-based lead-free solders, Microelectronics Reliability, Vol. 42, No. 6 (2002), pp. 951-966.

(2) M. Mukai, T. Kawakami, Y. Hiruta, K. Takahashi, K. Kishimoto, and T. Shibuya, Fatigue Life Estimation of Solder Joints in SMT-PGA Packages, Journal of Electronic Packaging, Transactions of the ASME, Vol. 120, No. 2 (1998), pp. 207-212.

(3) T. S. Park, and S. B. Lee, Low Cycle Fatigue Testing of Ball Grid Array Solder Joints under Mixed-Mode Loading Conditions, Journal of Electronic Packaging, Transactions on the ASME, Vol. 127, No. 3 (2005), pp. 237-244.

(4) I. Kim, and S. B. Lee, Development of a Novel Microimpact-Fatigue Tester and Its Application to Impact Reliability of Lead-Free Solder Joints, IEEE Transactions on Components and Packaging Technologies, Vol. 32, No. 3 (2009), pp. 542-549.

(5) C. H. Chen, S. X. Zhang, S. W. Ricky Lee, and L. Mohamed, Investigation on copper diffusion depth in copper wire bonding, Microelectronics Reliability, Vol. 51, No. 1 (2011), pp. 166-170.

(6) E. Stellrecht, B. Han, M. G. Pecht, Characterization of Hygroscopic Swelling Behavior of Mold Compounds and Plastic Packages, IEEE Transactions on Components and Packaging Technologies, Vol. 27, No. 3 (2004), pp. 499-506.

(7) S. K. Ryu, K. H. Lu, X. Zhang, J. H. Im, P. S. Ho, and R. Huang, Impact of Near-Surface Thermal Stresses on Interfacial Reliability of Through-Silicon Vias for 3-D Interconnects, IEEE Transactions on Device and Materials Reliability, Vol. 11, No. 1 (2011), pp. 35-43.

(8) L. J. Ladani, Numerical analysis of thermo-mechanical reliability of through silicon vias (TSVs) and solder interconnects in 3-dimensional integrated circuits, Microelectronic Engineering, Vo. 87, No. 2 (2010), pp. 208-215.

(9) M. Koganemaru, T. Ikeda, N. Miyazaki, Residual stress evaluation in resin-molded IC chips using finite analysis and piezoresistive gauges, Microelectronics Reliability, Vol. 48, No. 6 (2008), pp. 923-932.

(10) T. Ikeda, W. K. Kim, and N. Miyazaki, Evaluation of the Delamination in a Flip Chip Using Anisotropic Conductive Adhesive Films Under Moisture/Reflow Sensitivity Test, IEEE Transactions on Components and Packaging Technologies, Vol. 29, No. 3 (2006), pp. 551-559.

(11) T. Ikeda, I. Arase, Y. Ueno, N. Miyazaki, N. Ito, M. Nagatake, and M. Sato, Strength Evaluation of Plastic Packages During Solder Reflow Process Using Stress Intensity Factors of V-Notch, Journal of Electronic Package, Transactions of the ASME, Vol. 125, No. 1 (2003), pp. 31-38.

(12) J. Y. Yoon, I. Kim, and S. B. Lee, Measurement and Characterization of the Moisture-Induced Properties of ACF Package, Journal of Electronic Packaging, Transactions of 
the ASME, Vol. 131, No. 2 (2009), pp. 0210121-0210128.

(13) S. B. Lee, T. S. Park, and S. J. Ham, Experimental Techniques for Fatigue Reliability of BGA Solder Bumps in Electronic Packaging, JSME International Journal, Series A: Mechanics and Material Engineering, Vol. 43, No. 4 (2000), pp. 400-407.

(14) S. J. Ham, and S. B. Lee, Measurement of Creep and Relaxation Behaviors of Wafer-Level CSP Assembly Using Moiré Interferometry, Journal of Electronic Packaging, Transactions of the ASME, Vol. 125, No. 2 (2003), pp. 282-288.

(15) S. Y. Yang, W. S. Kwon, and S. B. Lee, Chip warpage model for reliability prediction of delamination failures, Microelectronics Reliability, Vol. 52, No. 4 (2012), pp. 718-724.

(16) J. H. Park, K. W. Jang, K. W. Paik, and S. B. Lee, A Study of Hygrothermal Behavior of ACF Flip Chip Packages With Moiré Interferometry, IEEE Transactions of Components and Packaging Technologies, Vol. 33, No. 1 (2010), pp. 215-221.

(17) J. W. Jang, J. H. Park, and S. B. Lee, In-Plane Deformation Measurement of Thin Packages Using an Atomic Force Microscope Moiré Method With a Pseudo-Phase-Shifting Technique, IEEE Transactions on Components, Packaging and Manufacturing Technology, Vol. 2, No. 12 (2012), pp. 1992-2000.

(18) J. W. Jang, and S. B. Lee, Thermally Induced Deformation Measurement of Through-Silicon Via (TSV) Structures using an Atomic Force Microscope (AFM) Moiré Method, IEEE Nano 2012 - 12th International Conference on Nanotechnology, Birmingham, UK, 2012. 